\title{
Transatlantica
}

Revue d'études américaines. American Studies Journal

\section{Le mythe de la Wilderness dans The Scarlet Letter}

\section{Lauric Guillaud}

\section{(2) OpenEdition}

Journals

Édition électronique

URL : http://journals.openedition.org/transatlantica/1581

DOI : 10.4000/transatlantica. 1581

ISSN : 1765-2766

Éditeur

AFEA

\section{Référence électronique}

Lauric Guillaud, «Le mythe de la Wilderness dans The Scarlet Letter», Transatlantica [En ligne], 1 | 2007, mis en ligne le 03 août 2007, consulté le 29 avril 2021. URL : http://journals.openedition.org/ transatlantica/1581; DOI : https://doi.org/10.4000/transatlantica.1581

Ce document a été généré automatiquement le 29 avril 2021

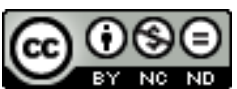

Transatlantica - Revue d'études américaines est mis à disposition selon les termes de la licence Creative Commons Attribution - Pas d'Utilisation Commerciale - Pas de Modification 4.0 International. 


\title{
Le mythe de la Wilderness dans The Scarlet Letter
}

\author{
Lauric Guillaud
}

1 Wilderness... Un mot-piège qui résume l'Amérique dans la complexité de son espacetemps, un mot-pieuvre dont la polysémie tentaculaire renvoie au tabou ultime: l'ensauvagement du corps et de l'âme. Au bout du chemin gît le bewilderment, mélange d'ahurissement, de perplexité, de confusion qui s'empare du civilisé désorienté. Menace de contagion, d'indianisation, de plongée régressive dans les abîmes des sylves obscures.

2 La wilderness, écrit J. R. Rougé, est « la réalité concrète d'une terre non encore explorée, secrète, inquiétante et dangereuse à la fois", et en même temps une "wilderness archétypale et spirituelle $»^{1}$. La wilderness, selon Marc Amfreville, est cette "Terre primitive et espace de solitude adamique, désert minéral et biblique sur lequel règne le Mal, mais aussi étendue ténébreuse de la selva où le héros, lancé à la poursuite de son double, retrouve le sauvage en lui-même $»^{2}$.

3 Archétypale, elle est souvenir instinctif de la terre du mythe, la terrifiante « waste and howling wilderness ", terre des animaux sauvages, "forêt de la nuit » originelle d'où montent les peurs archaïques et les élans des origines, chaos où l'on perd ses repères familiers. Mais c'est aussi la wilderness biblique, lieu de rédemption en même temps que de souffrance, "a place of tryall» [« un lieu d'épreuve»], comme le dit Thomas Shepard ${ }^{3}$. Cotton Mather, qui prédit le proche avènement du Christ en Nouvelle Angleterre, avertit ses condisciples de la présence de "Dragons» ou de "Meutes de Démons » dans la wilderness : "Le désert que nous traversons pour atteindre la Terre promise est plein de serpents volants de feu $»^{4}$.

4 L’Américain, comme le fait remarquer R. H. Pearce, "a généralement trouvé une définition de sa civilité par le biais de la définition de son contraire, la sauvagerie $»^{5}$. Les Indiens, «hommes sauvages, féroces et dégénérés» (Samuel Purchas) ou "émissaires de Satan» (Thomas Hooker), sont eux-mêmes assimilés à l'espace diabolique qu'ils occupent, d'autant que les récits de captivité ne cessent d'associer en une même terreur les indigènes et leur environnement menaçant. Les Puritains sont 
hantés par l'idée d'un espace païen, l'équivalent physique d'un chaos mental, au point que Michael Wigglesworth baptise l'Amérique du Nord la région de "l'éternelle nuit » et de la « mort menaçante " .

A waste and howling wilderness,

Where none inhabited

But hellish fiends, and brutish men

That Devils worshipped.

This region was in darkness plac't

Far off from heavens light,

Amidst the shadows of grim death

And of eternal night ${ }^{7}$

Le monde vierge est perçu comme le royaume de l'Antéchrist, et ses habitants, comme des «hommes transformés en bêtes» ou des «esclaves nus de Satan $»^{8}$. Au satanisme s'ajoute le sadisme. La chronique de William Bradford (of Plymouth Plantation, 1630-1651), premier historien américain, constitue un catalogue impressionnant des calamités du Nouveau Monde : «[...] ...la seule chose qu'ils voyaient était un paysage sauvage hideux et désolé, rempli d'hommes et de bêtes sauvages - et ils ne savaient évaluer ces multitudes $"$. Bradford insiste plus loin sur les dangers du contact avec les Indiens, «peuple sauvage, cruel, barbare et perfide, [...] non contents de tuer ou de prendre la vie, [les Indiens] se plaisent à tourmenter les hommes de la manière la plus sanglante qui soit $»$.

6 Le tableau des « grand-peurs indiennes » (Indian scares) est éloquent. Il s'agit d'affronter « ces créatures semblables aux bêtes par les instincts et les moeurs» (1660), de mener une «lutte à mort contre la bête en son repaire » (1675) ou de "reconnaître, tuer et détruire » $(1697)^{10}$. Autour d'un même centre de nuit gravitent des forces démoniaques ou bestiales qu'il faut annihiler en un combat sacré. "Mon Dieu, s'exclame Edward Taylor, contemple ce terrible ennemi dont les assauts farouches me font trembler ».

7 D'où les innombrables avertissements lancés à ceux qui ne craignent pas l'aliénation - le contact avec les indigènes «barbares et païens ». Partout, les ministres du culte brandissent la menace de «l'infection» [bewitchment] qui, dans leur esprit, correspond au mariage impie avec le Diable, double transgression religieuse et sexuelle. La wilderness est ce lieu de mort et de perdition où se mêlent condamnation spirituelle et dangers physiques. C'est surtout un espace fondamentalement ambivalent, jardin et anti-jardin à la fois, où l'on peut tout aussi bien retrouver l'innocence primitive que l'ultime sauvagerie, ce que les Puritains appellent «l'Eucharistie noire ${ }^{11}$. C'est ce paradoxe que l'on trouvera au centre même de la culture schizoïde américaine, inaugurée par les calvinistes.

8 Les récits d'enlèvement évoquent ces craintes de l'union avec «L'Homme Noir » de la forêt, peur et tentation à la fois de l'interdit, qu'on retrouve dans les affaires de sorcellerie. Dans cette wilderness spectrale, Mather et ses condisciples n'ont aucun mal à établir un lien organique entre les Indiens, le Diable et les hérétiques. Pour les Puritains, la religion indienne est manifestement une forme de sorcellerie. «Les sorciers indiens, écrit R. H. Pearce, participaient clairement aux merveilles du monde invisible $»^{12}$.

9 Cette paranoïa sera fatale à la théocratie puritaine, mais féconde en images gothiques qui irrigueront l'imaginaire américain. La Lettre écarlate de Hawthorne exprime à merveille la dualité d'une wilderness, à la fois source de liberté et lieu de tentation irrésistible - « the wilderness-temptation », écrivait John Eliot. 
10 Avant de cerner l'ambiguïté de la wilderness dans The Scarlet Letter, voyons comment la figure de l'Indien marque le roman de sa présence discrète et néanmoins prégnante. Il faut d'abord noter que les seuls Indiens décrits par Hawthorne le sont à l'intérieur de l'enceinte du settlement. D'une manière symétrique, on les retrouve dans les deux scènes de pilori qui ouvrent et concluent le roman. Paradoxalement, ils sont absents des scènes se déroulant dans la wilderness, mais leur présence est marquée symboliquement par l'assimilation graduelle d'Hester à leur statut de naturels et de parias.

11 Dans le troisième chapitre, l'Indien sert à introduire le personnage de Chillingworth. Le regard d'Hester passe d'une manière significative de l'Indien à son voisin.

From this intense consciousness of being the object of severe and universal observation, the wearer of the scarlet letter was at length relieved, by discerning, on the outskirts of the crowd, a figure which irresistibly took possession of her thoughts. An Indian, in his native garb, was standing there; but the red men were not so infrequent visitors of the English settlements, that one of them would have attracted any notice from Hester Prynne, at such a time; much less would he have excluded all other objects and ideas from her mind. By the Indian's side, and evidently sustaining a companionship with him, stood a white man, clad in a strange disarray of civilized and savage costume ${ }^{13}$.

12 L'hybridité de l'habillement indique non seulement l'introduction d'une hétérogénéité dans l'orthodoxie puritaine, mais une notion de passage ou de frontière dont l'importance sera attestée par la situation d'exilée d'Hester et de sa fille, à la lisière de la ville et de la forêt. On passe ainsi « des confins de la foule » à la « limite de la ville ", topologie fondamentale qui renvoie à la fois à l'opposition puritaine civilisation/ wilderness et au statut d'entre-deux qui caractérise Hester et l'Indien. Chillingworth luimême, fantôme revenu du passé comme des terres lointaines, qui entend "planter sa tente " ici, «sur ces sauvages confins de la terre» [" on this wild outskirt of the earth »], revendique lui aussi une situation d'entre-deux. Mais lui s'installe dans le cœur du settlement comme dans le cœur de ses futures proies. Avant même le début de l'action se dégage une topologie au centre de laquelle rayonne la wilderness. Dans la vision manichéenne des Puritains, la forêt s'associe à la sauvagerie et à la diablerie, de même que la cité représente à la fois Dieu et la civilisation. D'où l'importance du passage de l'une à l'autre, considéré comme une trans-gression au sens propre et au sens figuré. A l'instar d'Anne Hutchinson, Hester est bannie de la cité pour sa faute, tandis que son mari réintègre le giron puritain, non sans avoir passé plusieurs années dans la clandestinité de la wilderness. Ces deux passages symétriques, de la lumière aux ténèbres, et des ténèbres à la lumière, sont d'autant plus remarquables chez Hawthorne qu'ils contredisent les valeurs symboliques des deux topoi, comme nous le verrons.

Chillingworth est l'émanation d'un wild conforme aux normes puritaines. Les Puritains, en quête de paysage spirituel, cherchent dans la nature les «ombres des choses divines ", pour reprendre la formule de Jonathan Edwards, façonnant ainsi une sombre fantasmatique du décor. A force de traquer l'ordre divin dans la nature, ils sombrent dans une double peur obsédante : celle du péril de l'absence de Dieu et de la présence terrifiante du mal ${ }^{14}$. Polarisés par l'ordre social, ils finissent par succomber à la crainte permanente du chaos : peur de la colère divine, du délitement communautaire. Quand un serpent s'introduit dans la salle du Synode à Cambridge, en 1648, Winthrop note dans son journal : «Le serpent, c'est le Diable ». Le serpent biblique sort de la Genèse 
pour s'insinuer dans la cité de Dieu, tel un signe funeste. Le difforme Chillingworth se glisse lui aussi dans la cité tel un serpent:

A writhing horror twisted itself across his features, like a snake gliding swifthy over them, and making one little pause, with all its wreathed intervolutions in open sight. [...] After a brief space, the convulsion grew almost imperceptible, and finally subsided in the depths of his nature. 45

Cet homme impénétrable qui revient en terre chrétienne est en fait une créature du mal qui tente de sauver les apparences en expliquant son étrange absence par un séjour involontaire chez les Indiens.

I am a stranger, and have been a wanderer, sorely against my will. I have met with grievous mishaps by sea and inland, and have long been held in bonds among the heathenfolk, to the southward; and am now brought hither by this Indian, to be redeemed out of my captivity. 45

L'auteur exploite ici le filon populaire des récits de captivité, le plus célèbre étant celui écrit par Mary Rowlandson, emmenée prisonnière en 1676 sur la frontière du Massachusetts : «[I went] mourning and lamenting, leaving farther my own Country, and travelling into the vast and howling Wilderness $»^{15}$. Le destin de Mary illustre la terreur de la chute, de la dégénérescence - le mariage blasphématoire avec la nature sauvage et ses habitants dépravés, l'infection de ou par l'indianisation. Derrière la terreur perce aussi la tentation de l'abandon ou de la transgression. Tous ces doutes pèsent aussi sur Chillingworth. Doutes d'autant plus légitimes que lui et l'Indien semblent en connivence, se comprenant même (46). Chillingworth l'alchimiste a manifestement développé ses connaissances au contact des habitants de la wilderness.

In his Indian captivity, moreover, he had gained much knowledge of the properties of native herbs and roots; nor did he conceal from his patients, that these simple medicines, Nature's boon to the untutored savage, had quite as large a share of his own confidence as the European pharmacopeiae, which so many learned doctors had spent centuries in elaborating. 80

L'obscurantisme se conjugue ainsi aux pratiques obscures des sorciers indiens, renforçant dans tous les cas la valorisation ténébreuse de la forêt et de sa magie noire.

Two or three individuals hinted that the man of skill during his Indian captivity, had enlarged his medical attainments by joining in the incantations of the savage priests, who were universally acknowleged to be powerful enchanters, often performing seemingly miraculous cures by their skill in the black art. 85

Les thérapies que propose Chillingworth, baptisé « Homme Noir » par Pearl, ne peuvent que susciter la méfiance chez Hester: "I have learned many new secrets in the wilderness, and here is one of them,--a recipe that an Indian taught me, in requital of some lessons of my own, that were as old as Paracelsus » (52). Il y a bien là mariage blasphématoire entre deux formes de sorcellerie, l'une de l'Ancien Monde, l'autre du Nouveau. Et ce « medicine-man » qui n'ose dire son nom revient distiller, voire instiller ses potions païennes, dans les organismes purs des habitants de la cité, de même qu'il va contaminer peu à peu le Révérend Dimmesdale. Si la potion du Malin exerce une action positive sur Pearl, c'est que cette enfant de la forêt est en sympathie avec son environnement sauvage et païen. On ne peut en dire autant de Dimmesdale. Celui-ci décline jour après jour, devenant aussi pâle que Chillingworth gagne en noirceur, comme si se déroulait quelque étrange mutation alchimique d'un organisme à l'autre, comme si l'homme noir déversait chaque jour dans les veines de Dimmesdale son poison issu des herbes nocives de la wilderness. Celle-ci, à l'image des croyances 
puritaines, finit ainsi par infecter véritablement le settlement, en la personne de son Révérend.

Voyons maintenant les effets du nouvel environnement d'Hester, « on the outskirts of the town ", là où la loi puritaine l'a exilée, à l'écart de la cité de Dieu. Comme le remarque Simone Pellerin, sa situation se rapproche de celle de l'Indien:

...l'obligation imposée à Hester Prynne de vivre à la marge de la société, aux limites de la ville de Boston, rappelle la place où se tient l'Indien qui accompagne son «prisonnier ». Hester est au bord de la ville comme cet Indien anonyme, donc générique. Quoi d'étonnant alors à ce que, lorsque son esprit vagabonde, elle entre dans un état de "sauvagerie » ? La compréhension qu'a Hester du fonctionnement de Boston et du puritanisme la met dans la situation de l'Indien. Si elle doit être en contact avec la société "civilisée », elle est à la marge. Si elle n'y est pas obligée, alors elle erre, comme parfois le font « son esprit et son cœur », en toute liberté, tel l'Indien dans sa forêt. La rigueur de ce puritanisme, qui est la forme de société «pure » produite par l'Europe, ne peut exister dans sa pureté revendiquée qu'aux marges mêmes du monde occidental. Elle lui est aussi marginale que l'Indien venu accompagner un captif et observant la scène du pilori ${ }^{16}$.

La situation d'Hester, paria dans le settlement, se retrouve matérialisée dans la wilderness. S'étant mise en marge de la loi, elle vit au bord du monde civilisé mais un changement radical s'opère en elle, dû à la proximité d'un monde qui la contraint à adopter le point de vue des habitants de la forêt. Hawthorne insiste sur l'opposition croissante qui s'affirme sur le plan spatial et topologique entre des mondes qui gravitent indépendamment les uns des autres. Le discours sur le bord se double d'un discours sur les sphères: "Mother and daughter stood together in the same circle of seclusion from human society 》 (65); « ...was there a circle of ominous shadow moving along with his deformity?» (114); " [Hester and Dimmesdale] now felt, at least, inhabitants of the same sphere » (123); « within the magic circle of this hour » $(130) ;$ « ... so remote from her own sphere » (154); « ...her whole orb of life » (155); « Hester stood in that magic circle of ignominy...» (156). Autant d'isolats de solitude ou d'exil physiques et moraux qui expriment des espaces de rejet, ainsi que le malaise qui court dans la société puritaine.

En se tenant à l'extérieur du settlement, Hester est véritablement une outsider, séparé des insiders, les colons qui vivent dans l'enceinte sacrée et perçoivent les présages et les menaces des puissances de l'ombre. Elle se confond avec les créatures du monde extérieur, univers hostile où conspirent à la fois démons, sorcières, pécheurs et hérétiques, maudits et bannis, tels les Quakers, ces dangereux outsiders qui fréquentent la Frontière pour mieux propager leur hérésie démoniaque. Il fallut, paradoxalement, de nouveaux exclus pour que la liberté avance en Amérique du Nord. Le schisme religieux et le désir d'utopie vont bouleverser le paysage nord-américain en quelques années, de Thomas Hooker à William Penn, en passant par Roger Williams et Anne Hutchinson, première femme à défier les autorités de la Colonie de la Baie et à être condamnée à l'exil et excommuniée par l'Église de Boston. Une femme a donc réussi à ébranler les fondations politiques et religieuses des puritains. Étrange époque, peutêtre unique dans l'histoire, où un schisme peut créer les conditions géopolitiques d'une indépendance territoriale. Tous ces dissidents ont laissé passer un peu d'air pur dans la «maison» puritaine. L'uniformité puritaine ne résistera pas longtemps aux coups de boutoir des « ultra-orthodoxes", des rebelles, ou des tenants de la " voie de l'ombre». Ce n'est pas un hasard si Hester est comparée à Anne Hutchinson (Ch. XIII). Dans la solitude de son supplice, Hester renonce « aux règles, aux principes et aux préjugés de 
la religion » et acquiert " l'esprit critique », comme le note Jacques Cabau ${ }^{17}$, au terme d'une évolution décrite ainsi par Hawthorne :

Hester Prynne, with a mind of native courage and activity, and for so long a period not merely estranged, but outlawed, from society, had habituated herself to such latitude of speculation as was altogether foreign to the clergyman. She had wandered, without rule or guidance, in a moral wilderness ; as vast, as intricate and shadowy, as the untamed forest, amid the gloom of which they were now holding a colloquy that was to decide their fate. Her intellect and heart had their home, as it were, in desert places, where she roamed as freely as the wild Indian in his woods. For years past she had looked from the estranged point of view at human institutions, and whatever priests or legislators had established; criticizing all with hardly more reverence than the Indian would feel for the clerical band, the judicial robe, the pillory, the gallows, the fireside, or the church. The tendency of her fate and fortunes had been to set her free. The scarlet letter was her passport into regions where other women dared not tread. 128

21 De cette citation stratégique se dégagent trois concepts fondamentaux reliés les uns aux autres: la liberté, l'Indien et la wilderness. En un bond historique révélateur, Hawthorne quitte l'obscurantisme du $17^{\mathrm{e}}$ siècle pour le siècle des Lumières, évoquant le droit naturel et le «bon sauvage ». La nature sauvage est réhabilitée par la figure de l'Amérindien, réappropriée, faut-il le rappeler, par les Insurgés des colonies pour symboliser la liberté naissante à la fin du $18^{\mathrm{e}}$ siècle. Hester se présente ici comme une "prophétesse» des temps futurs, comme l'avait suggéré Hawthorne (108), venant annoncer du fond de sa retraite sylvestre de nouvelles perspectives, plus humaines et plus rationnelles. Grâce à sa faute originelle, " passeport » pour l'exil, elle acquiert une supériorité morale et intellectuelle qui lui est conférée par son statut même de bannie, d' "étrangère » comme le précise l'auteur. La wilderness est ainsi revalorisée, inversée même, comme Hester sublime sa faute. A l'image de la lettre écarlate changeant de couleur dans la forêt, elle se métamorphose en ce lieu sauvage, topos même d'une altérité quasi surnaturelle : «Her mind, and especially her memory, was preternaturally active, and kept bringing up other scenes than this roughly hewn street of a little town, on the edge of the Western wilderness " (43). Le wild, cette antichambre de l'enfer, est ici l'antithèse même de la loi puritaine, offrant même un refuge à ses victimes. Ce sanctuaire païen littéralement hors la loi [outlaw], dans lequel est rejetée Hester hors du settlement [outcast], la place tout naturellement au côté des Indiens : « ...and having also the passes of the dark, inscrutable forest open to her, where the wildness of her nature might assimilate itself with a people whose customs and life were alien from the law that had condemned her " (56). Sa fille n'est pas en reste: «Pearl was a born outcast of the infantile world. An imp of evil, emblem and product of sin, she had no right among christened infants » (64). C'est au sein de la nature païenne que Hester est régénérée et transfigurée, dans un "flot de lumière ensoleillée ", telle un[e] Noble Sauvage, "the wildest Indian of them all! ", s'exclamera Leslie Fiedler ${ }^{18}$. Tout s'inverse, à l'image de la colonie puritaine désormais décrite comme "si lointaine et si obscure » et d'une wilderness qui offre maintenant à Hester "l'étrangère " une " seconde naissance " dans une seconde " patrie ", " une patrie sauvage et lugubre mais où elle était véritablement chez elle pour toute la vie" [lifelong home]. On peut ainsi se demander, avec Jacques Cabau, si, loin de grandir en sainteté, Hester ne grandit pas «au contraire en diabolisme » (137).

Tout se passe comme si Hester, dans ce lieu consacré, avait vécu une initiation, affrontant les périls d'une wilderness intériorisée, et traduite par des métaphores 
archétypales qui renvoient à Charles Brockden Brown : « ...Hester Prynne, whose heart had lost its regular and healthy throb, wandered without a clew in the dark labyrinth of mind; now turned aside by an insurmountable precipice; now starting back from a deep chasm » (108). Dotée « d'un sens nouveau », Hester adopte peu à peu le point de vue de l'Indien et retrouve dans le « forestland » une nouvelle pureté en respirant l'air « d'une région libre, non régénérée, non christianisée, encore sans loi » (129). Une pureté qui, cela va sans dire, s'oppose au puritanisme répressif et ténébreux, et qui est isomorphe d'une lumière qui irradie la nature en une apothéose de rédemption païenne, «a mystery of joy » écrit Hawthorne (130). Nouvelle inversion spectaculaire en ce chapitre intitulé "A Flood of Sunshine ", la forêt se retrouve libre de toute connotation sombre ou diabolique, offrant son sanctuaire à Hester et à Dimmesdale, miraculeusement réunis dans ce lieu discret, "nature sauvage et païenne de la forêt » qui marque sa "sympathie à ces deux esprits inondés de bonheur ». Une sympathie qu'emblématise excellemment Pearl, « the elf-child », qui semble le rejeton même de la wilderness : « ... the mother-forest and these wild things which it nourished, all recognized a kindred wildness in the human child» (131). Une enfant qui court la forêt à l'instar de ses habitants ténébreux, pourchassant en hurlant la blanche progéniture de la cité (69).

L'une des transformations les plus spectaculaires, consécutives au passage dans la wilderness, s'opère chez le Révérend Dimmesdale. Dans ce sanctuaire naturel triomphent la non-conformité et l'ouverture, loin de la clôture puritaine et de ses inhibitions. Là, tombent les masques moraux et sociaux. Dimmesdale parle ouvertement pour la première fois et exprime ouvertement ses émotions dans ce lieu de liberté recouvrée, de même qu'Hester n'hésite plus à révéler ses pensées à son amant, devenue ici son égale, au point de lui parler, nouvelle inversion, comme un Révérend à ses ouailles : « Begin all anew! [...] Preach! Write! Act! » (127) Hester la « sauvage » n'oublie pas d'associer la topologie à son sermon libertaire :

Whither leads yonder forest track? Backwards to the settlement, thou sayest! Yes; but onward, too! Deeper it goes, and deeper, into the wilderness, less plainly to be seen at every step! until, some few miles hence, the yellow leaves will show no vestige of the white man's tread. There thou art free! 127

D'une manière significative, Hester encourage Dimmesdale à tourner le dos au settlement et à devenir, tel John Eliot, «le guide et l'apôtre des Peaux-rouges » (127). Même si le Révérend Dimmesdale décide finalement de réintégrer la cité, il s'opère chez lui une transformation intéressante qui va précipiter la fin de l'histoire. Si Chillingworth est infecté négativement par les pratiques ténébreuses des habitants de la wilderness, Dimmesdale, quant à lui, est transfiguré par son séjour dans les lumières de la forêt, ressentant même les premiers symptômes de la rébellion : « ...he was incited to do some strange, wild, wicked thing or other, with a sense that it would be at once involuntary and intentional... "(p.139). Sorti du wild, le pasteur est «bewildered» (184), victime d'un étrange pouvoir que possède d'ailleurs Pearl, l'enfant sauvage (" this bewildering and baffling spell », 64). Il éprouve des sensations nouvelles, comme s'il avait laissé derrière lui dans la forêt les oripeaux du vieil homme pour affirmer une nouvelle identité : «I am not the man for whom you take me! I left him yonder in the forest, withdrawn into a secret dell, by a mossy tree-trunk, and near a melancholy brook!» (138) La rencontre avec Hester au sein de la forêt a libéré Dimmesdale, lui donnant un surcroît d'énergie surnaturelle qui lui permettra de faire triompher la vérité au cœur du settlement, lieu même de la dissimulation. 
me en écho au chapitre II, on retrouve au chapitre XXII des Indiens sur la Place du Marché, au pied du pilori, comme si ce chœur grec insolite accompagnait le drame qui se noue. Muets et quasiment invisibles, ils semblent figés dans une gravité solennelle, comme s'ils assistaient à la fin d'un monde, le leur et celui des Puritains. La virevoltante Pearl bouscule le décorum, confirmant pour la dernière fois son statut de naturelle. Si sa mère est devenue mentalement une Indienne au contact de la nature, Pearl, elle, semble l'avoir toujours été :

She ran and looked the wild Indian in the face; and he grew conscious of a nature wilder than his own. Thence, with native audacity, [...] she flew into the midst of a group of mariners, the swarthy-looking wild men of the ocean, as the Indians were of the land; and they gazed wonderingly and admiringly at Pearl, as if a flake on the sea-foam had taken the shape of a little maid, and were gifted with a soul of the seafire that flashes beneath the prow in the nighttime. 155

On s'aperçoit que les principaux protagonistes du roman sont transformés par leur passage dans la wilderness, avec parfois des conséquences ironiques ou cruelles. Si Hester est rédimée par la nature sauvage, sa fille, qui en semble l'émanation même, finira par devenir une riche héritière à la mort de sa mère. Dimmesdale, transfiguré par le monde de la forêt, rejoint l'enfer puritain pour y mourir en martyr, tandis que Chillingworth, après avoir usé et abusé des drogues de la wilderness, se dessèche telle une feuille morte sans avoir pu assouvir sa vengeance.

L'œuvre de Hawthorne s'ancre dans un double espace-temps historique qui montre l'évolution significative du mythe de la wilderness. Si l'auteur est fidèle à la représentation maléfique de la nature sauvage par ses ancêtres puritains, il rédige son roman en 1850, à une époque où commence à s'imposer le mythe de la Frontière, source de liberté pour les futurs pionniers. Hester tente de persuader Dimmesdale de fuir vers l'Ouest pour devenir ainsi un vrai Américain. «Doth the universe lie within the compass of yonder town? [...] There thou art free! Is there not shade enough in all this boundless forest to hide thy heart from the gaze of Roger Chillingworth? » Dimmesdale, typique Puritain, ne peut que répondre mélancoliquement: "Yes, Hester, but only under the fallen leaves » (127). Comme le souligne Leslie Fiedler, " pour lui, la route de l'Ouest, est un chemin interdit qui ne mène nulle part, sinon à la damnation et la mort $»^{19}$. Si les exhortations d'Hester (« Begin all anew! », 127 ; « Let us not look back, [...] the past is gone »,130) ne trouvent guère d'écho chez son amant, elles expriment un nouveau credo national, lié à l'expansion et à la Frontière. L'ambiguïté de la wilderness est donc totale chez Hawthorne : la forêt est le royaume des sorcières, de l'illégalité, de l'immoralisme. Mais c'est surtout le lieu de la rédemption d'Hester et de son envol vers les sphères de l'intellect et de la liberté. Hester incarne ainsi, non seulement la transformation de l'individu en personne éclairée, mais celle de l'espace qui lui est consubstantielle. Après le temps de la wilderness calviniste, voici le temps des "grands espaces «(«open spaces»). En sortant de la cité, Hester est sortie de la Loi pour trouver dans la redoutable nature les conditions de son émancipation. La liberté a toujours quelque chose de monstrueux. « La vraie liberté est noire », dira Artaud.

Hawthorne a bien compris que le véritable débat se situe à la frontière, dans cet "entre-deux» où s'affrontent deux conceptions de l'Amérique, dans cet espace périphérique mouvant que privilégie l'écrivain. Il y trouve en effet l'union idéale du réel et de l'imaginaire, des "Frontières » extérieure et intérieure que l'Amérique s'acharne à concilier depuis toujours, afin de découvrir la couleur mystérieuse de la « lettre », c'est-à-dire des « hiéroglyphes » de la wilderness. 


\section{NOTES}

1. Jean-Robert Rougé, « Les significations des frontières dans l'histoire américaine ", Frontière et frontières dans le monde anglophone (Paris : Presses Universitaires de ParisSorbonne, 1991), 14.

2. Marc Amfreville, Charles Brockden Brown (Paris : Belin, 2001), 30.

3. Cités par Peter N. Carroll, Puritanism and the Wilderness (New York : Columbia University Press, 1969), 65 et 70.

4. Cité par Mircea Eliade, La Nostalgie des origines (Paris : Gallimard, 1971), 158.

5. Roy Harvey Pearce, cité par Elise Marienstras, Les mythes fondateurs de la nation américaine (Paris : Ed. Complexe, 1992), 159.

6. Cité par Roderick Nash, Wilderness and the American Mind (New Haven : Yale University Press, 1967), 36.

7. Michael Wigglesworth, « God's Controversy with New England », Early American Writing (New York : Penguin, 1994), 209.

8. Alexander Whitaker « Good Newes from Virginia », Early American Writing, 105 ; J. White (1630), cité par Jean-Pierre Martin, Le Puritanisme américain en Nouvelle-Angleterre (Pessac : P. U. de Bordeaux, 1989), 58.

9. Cité par Charles Berryman, From Wilderness to Wasteland (National University Publications, Port Washington, N.Y. : Kennikat Press, 1979), 6.

10. Voir Jean-Pierre Martin, Le Puritanisme américain en Nouvelle-Angleterre, 59.

11. Voir Richard Slotkin, Regeneration Through Violence (Middletown, CT : Wesleyan U. P., 1973), 99.

12. R. H. Pearce, Savagism and Civilization (Baltimore, MD : Johns Hopkins Press, 1969), 22.

13. Nathaniel Hawthorne, The Scarlet Letter and Other Writings, L. S. Person ed. (Norton Critical Edition, New York \& London : Norton, 2005), 44.

14. Voir notre ouvrage, La Terreur et le sacré (Paris : Michel Houdiard Editeur, 2003).

15. Citée par Roderick Nash, Wilderness and the American Mind, 28.

16. Simone Pellerin, « 'A subtlety so perfect', la substantielle transparence des 'naturels' dans The Scarlet Letter », dans The Scarlet Letter de Nathaniel Hawthorne, ouvrage dirigé par Marc Amfreville et Antoine Cazé (Paris : Ed. Ellipses, 2005),169.

17. Jacques Cabau, La Prairie perdue (Paris : Le Seuil, 1966), 135.

18. Leslie Fiedler, Love and Death in the American Novel (New York: Anchor, Doubleday, 1992), 443.

19. Leslie Fiedler, Le Retour du Peau-Rouge (Paris : Le Seuil, 1971), 110.

\section{AUTEUR}

\section{LAURIC GUILLAUD}

Université du Maine 